\title{
Changes in focal interictal epileptiform activity during and after the performance of verbal and visuospatial tasks in a patient with intractable partial seizures
}

\author{
S J Boniface, R P Kennett, J M Oxbury, S M Oxbury
}

\begin{abstract}
An 18-year-old male with intractable complex partial seizures is described in whom localised epileptiform discharges in the EEG were influenced in a specific manner by different cognitive tasks. The patient had impaired verbal skills but above average visuospatial ability, and seizures probably arising in the left temporal lobe. Comparison of verbal and visuospatial tasks showed that focal epileptiform activity was suppressed or enhanced depending on the nature of the immediate and preceding cognitive tasks. The finding of particular interest was the activity of a posterior temporal spike focus only during rest periods after verbal tasks, by contrast with an independent mid-to-anterior temporal focus that was suppressed during verbal tasks.
\end{abstract}

(F Neurol Neurosurg Psychiatry 1994;57:227-228)

The interaction between epilepsy and cognitive performance has been of interest since the late 1930s. Several studies have concentrated on generalised epileptiform abnormalities, but Binnie et al have shown that the occurrence of discharges localised to the left or right sides are associated with transient deficits in verbal and spatial tasks, respectively. ${ }^{1}$ Effects on the frequency of spontaneous epileptiform EEG discharges have been studied, with several authors showing a fall during cognitive activity ${ }^{2-5}$ and others suggesting a rise. ${ }^{6}$ There is also evidence suggesting that seizures can be induced by cognitive tasks mediated ipsilateral to the epileptogenic focus. ${ }^{7}$ We report a patient with left temporal lobe epilepsy and bilateral representation for language function in whom differing epileptiform discharges were suppressed or enhanced depending on whether the patient was assessed during or after the task, as well as on the nature of the task itself.

The patient was 18 years old. The pregnancy and perinatal period were normal and the first convulsion had occurred at 3 years of age one day after vaccination for measles. His habitual seizures started at the age of 5 and consisted of automatisms (180 per year on average) and secondarily generalised convulsions (about 18 per year). The automatisms lasted 2-3 minutes during which his arms would stiffen, he would lose contact with his surroundings, and utter obscenities. The convulsions were predominantly right sided but occasionally became generalised and were sometimes followed by a transient right hemiparesis with aphasia. Treatment was with carbamazapine and phenytoin. He was right handed and physical examination was normal. On neuropsychological assessment his verbal memory and expression skills were found to be poor, with a verbal IQ of 73. By contrast his visuospatial abilities were good with a performance IQ of 94 and a superior performance on constructional tasks equivalent to an IQ of 124. A carotid amytal test showed that representation for language (including spelling and naming) was bilateral.

Magnetic resonance imaging was suggestive of left hippocampal atrophy. Electroencephalography showed frequent multifocal spike and slow wave discharges at the left mid-to-anterior temporal scalp electrodes that were maximal at the sphenoidal electrode. Independent focal sharp waves also occurred in a similar distribution over the right temporal region with a frequency of less than 1 in 15 relative to the frequency of the left temporal sharp waves. These findings were considered to be consistent with a primary epileptogenic focus in the mesial structures of the left temporal lobe.

\section{Method}

The EEG was recorded during the performance of a total of four different verbal and visuospatial cognitive tasks. The verbal tasks were a picture vocabulary test (with simple objects from the Peabody picture vocabulary used as a naming test) and graded word spelling. The word spelling was based on the Schonell graded word spelling list supplemented by words of equivalent difficulty up to the patient's spelling limit of 9.5 years. The visuospatial tasks were an extended cube counting test ${ }^{8}$ and origami with pictorial instructions from a manual (origami was a hobby). The level of each task was selected according to the patient's abilities to minimise any effects dependent on the difficulty of the tasks rather than their nature. The tasks were performed once each for at least four minutes, interspersed by rest periods of 
Table Spike counts recorded at left and right temporal electrodes during the performance of four tasks

\begin{tabular}{lll}
\hline & \multicolumn{2}{c}{ Mean spike count (spikes per minute) } \\
\cline { 2 - 3 } Task & Left temporal & Right temporal \\
\hline Cube counting & 57 & 9 \\
Origami & 58 & 5 \\
Picture naming & 24 & 5 \\
Spelling & 28 & 2 \\
\hline
\end{tabular}

equivalent duration. During rest periods the subject remained alert with his eyes open and was allowed to relax from the task. Verbal and visuospatial tasks were in alternate order and rest periods were given at the beginning and end of the sequence.

\section{Results}

The EEG showed the left mid-to-anterior temporal spikes described, which continued throughout all the test conditions and rest periods at a frequency that was dependent on the cognitive task. An independent left posterior temporal spike focus was also found, which occurred exclusively during rest periods that followed verbal tasks (mean $56 /$ min for picture naming and $48 / \mathrm{min}$ for spelling). These posterior temporal discharges started within a minute of stopping the task and lasted for up to six minutes.

The mean left mid-to-anterior temporal spike count for the rest periods that were free of posterior temporal discharges showed little variation (range 54-59/min for all rest periods) and was comparable with the count during both of the visuospatial tasks (mean $57 / \mathrm{min}$ for cube counting and $58 / \mathrm{min}$ for origami; table). By comparison with visuospatial tasks, however, the mean left mid-toanterior temporal spike count was more than halved during verbal tasks (mean 24/min for picture naming and $28 / \mathrm{min}$ for spelling; ( $p<0.05$, Kruskal-Wallis ANOVA). By contrast, there was no significant change in the low rate of right-sided mid-to-anterior temporal discharges during the performance of tasks (range 2-9/min for all test conditions and rest periods, $\mathrm{p}>0.05$ )

\section{Discussion}

In a study by Kasteleijn-Nolst Trenité et al increased subclinical epileptiform discharges were shown to occur over both hemispheres during reading in children with partial or secondarily generalised epilepsy. ${ }^{9}$ The left-sided discharges, however, were increased to a lesser extent than those on the right. This was interpreted as evidence in support of the view that cognitive tasks cause relative suppression when they activate a region of the brain within the epileptogenic zone. Also, no significant differences occurred, in the discharge rates between the two hemispheres for various intelligence subtests, which included some verbal and spatial/perceptual cognitive tasks. By contrast our patient showed an absolute decrease in the frequency of left temporal discharges during verbal tasks, but not during visuospatial tasks, without an accompanying overall increase in right- and left-sided discharges. Paradoxically this suppressive effect may have been independent of the lateralisation for language function as there was evidence from carotid amytal testing that this was represented bilaterally in our patient.

Most relevant, as well as spontaneous midto-anterior discharges referable to the mesial structures of the left temporal lobe, cessation of verbal tasks was followed by the development for a short period of an independent ipsilateral posterior temporal spike focus. In studies of this type and in the clinical assessment of patients with focal seizures, therefore, factors precipitating the development of epileptiform features in the EEG during a period of rest may include the nature of the preceding cognitive activity. The provocation of occult localised EEG abnormalities during periods of rest after appropriate cognitive tasks may also find a role in the assessment of patients before surgery for epilepsy.

We are indebted to Mrs Anna Yalci for her technical assistance.

1 Binnie CD, Kasteleijn-Nolst Trenité DGA, Smit AM et al. Interactions of epileptiform EEG discharges and cognition. Epilepsy Res 1987;1:239-45.

2 Guey J, Tassinari CA, Charles C, et al. Variations du niveau d'efficience en relation avec des décharges épileptiques paroxystiques. Rev Neurol 1965;112:311-7.

3 Vidart L, Geier S. Enregistrements télé-encephalographiques chex des sujets epileptiques pendant le travail. Rev Neurol 1967;117:475-80

4 Hutt SJ, Newton S, Fairweather H. Choice reaction time and EEG activity in children with epilepsy. Neuropsychologia 1977;15:257-67.

5 Kasteleijn-Nolst Trenité DGA, Bakker DJ, Binnie CD, et al. Psychological effects of epileptiform EEG discharges. al. Psychological effects of epileptiform EEG

6 Wilkins AJ. On the manner in which sensory and cognitive processes contribute to epileptogenesis, and are disprocesses contribute to epileptogenesis, and are disrupted

7 Helmstaedter C, Hufnagel A, Elger CE. Seizures during cognitive testing in patients with temporal lobe epilepsy: Possibility of seizure induction by cognitive activation Epilepsia 1992;35:892-7.

8 Ratcliffe G. Aspects of disordered space perception. [Doctoral Thesis] Oxford: University of Oxford, 1970.

9 Kasteleijn-Nolst Trenité DGA, Siebelink BM, Berends SGC, van Strien JW, Meinardi H. Lateralized effects of sub-clinical epileptiform EEG discharges on scholastic performance in children. Epilepsia 1990;31:740-6. 